\title{
Immunohistochemical Expression of Fascin in Oral Epithelial Dysplasia and Oral Squamous Cell Carcinoma
}

\author{
Shilpa C Natesan ${ }^{1}$, Bindhu P Ramakrishnan ${ }^{2}$, Rekha Krishnapillai ${ }^{3}$, Priya Thomas ${ }^{4}$
}

\begin{abstract}
Aims: The present study aimed to evaluate and compare the expression of fascin in normal oral mucosa (NOM) and oral epithelial dysplasia (OED) and to estimate the expression in different grades of oral squamous cell carcinoma (OSCC).

Materials and methods: The study involves a total of 55 cases including 30 cases of OSCC, 15 cases of OED, and 10 NOM tissues. All the sections were subjected to fascin immunostaining. Epithelial dysplasia were scored by layer-wise immunohistochemical staining, while for carcinoma staining intensity and number of cells stained were assessed. The scores were analyzed using one-way ANOVA test and Tukey's post hoc test. Results: Our results showed that fascin expression in NOM was very low or restricted to the basal layers of the epithelium. In case of OED and OSCC, fascin immunostaining was highly elevated. Also, it was seen increasing with increasing grades of dysplasia $(p=0.002)$ and decrease in differentiation of OSCC.

Conclusion: A steady up-regulation of fascin expression is noted in OED and squamous cell carcinoma. These findings suggest that fascin plays an important role from early stages of carcinogenesis to invasive carcinoma.

Clinical significance: Fascin can be used as a reliable biomarker for diagnostic and prognostic implications of OED and OSCC, respectively. Keywords: Fascin, Head and neck cancer, Immunohistochemistry, Oral cancer, Oral epithelial dysplasia, Oral leukoplakia, Oral squamous cell carcinoma.

World Journal of Dentistry (2019): 10.5005/jp-journals-10015-1658
\end{abstract}

\section{INTRODUCTION}

Oral squamous cell carcinoma (OSCC) occurring in the head and neck region is the eleventh most common type of cancer in the world. ${ }^{1}$ However, in India there is a high incidence of oral cancer with tobacco being the most important causative factor. According to statistics by Takiar et al., the pooled projected score of head and neck cancers in India for the year 2020 is expected to be $218,421{ }^{2}$ Besides the advances in surgery, chemotherapy, radiation, and other combinational therapies, the prognosis of oral cancer has not improved in years.

Oral cancer is often preceded by potentially malignant disorders, which include oral leukoplakia, oral submucous fibrosis, erythroplakia, and lichen planus. ${ }^{3}$ Among them, oral leukoplakia is the most common with a malignant transformation risk of 1.5 to $34 \% .{ }^{4}$ The key to successful treatment outcome and survival rate of OSCC therefore depends on early diagnosis of these potentially malignant disorders. The gold standard method of diagnosis for oral epithelial dysplasia (OED) still remains to be the microscopic evaluation of hematoxylin-and-eosin stained sections. Histopathologically, all oral leukoplakias may not show epithelial dysplasia and many dysplastic lesions have shown to regress with time. ${ }^{5}$ Histopathological diagnosis of OED can lead to subjective errors in assessing dysplasia. Also, the fact that oral cancer being developed from lesions that lacked dysplastic changes have led many researchers to consider for reliable biomarkers in diagnosing OED. ${ }^{6}$

Fascin (FSCN1), a highly conserved $55 \mathrm{kDa}$ actin binding and bundling protein, is found to be up-regulated in many human carcinomas. It is primarily expressed in cells requiring motility like dendritic cells, neuronal cells, vascular endothelial cells, fibroblasts, and macrophages. ${ }^{7,8}$ This globular actin cross-linking protein plays an important role in the organization of cortical cell protrusions like filopodia, lamellipodial ribs, spikes, microvilli, and dendrites. ${ }^{8,9}$

\begin{abstract}
${ }^{1-4}$ Department of Oral Pathology and Microbiology, Annoor Dental College and Hospital, Muvattupuzha, Kerala, India

Corresponding Author:Shilpa CNatesan, Department of Oral Pathology and Microbiology, Annoor Dental College and Hospital, Muvattupuzha, Kerala, India, Phone: +91 9048605496, e-mail: sshilpacn@gmail.com
\end{abstract}

How to cite this article: Natesan SC, Ramakrishnan BP, Krishnapillai R, et al. Immunohistochemical Expression of Fascin in Oral Epithelial Dysplasia and Oral Squamous Cell Carcinoma. World J Dent 2019;10(5):340-345.

Source of support: Nil

Conflict of interest: None

For any carcinoma to spread, cancer cells need to acquire the ability to migrate. The progression of cancer cells are achieved by the active remodeling of actin cytoskeleton. Fascin organizes F-actin into parallel bundles and helps in the formation of these cellular protrusions. These protrusions have roles in cell-matrix adhesions, cell interactions, and migration. Also, they are rich in matrix metalloproteinases (MMPs), which when released, results in the degradation of extracellular matrices thus helping in the progression and invasion of carcinoma..$^{10,11}$

Metastasis of oral cancer is achieved by the migration of dysplastic epithelial cells after breaching the basement membrane. Though the exact molecular mechanism for cell migration is not elucidated, several theories explore the morphological polarization of cells, formation of membrane extensions like filopodia and lamellipodia and cell-substratum attachments. ${ }^{12}$ Whenever an external migratory stimulus becomes active, cells become anterioposteriorly polarized and extend protrusions in the direction of the signal. Cell-matrix adhesions attach these protrusions to the 
substratum on which the cell is migrating. These adhesions provide anchorage to the movement of cells and provide signals for the protrusive activity. ${ }^{13}$

Unlike other actin cross-linking proteins, fascin forms very tightly packed and ordered parallel F-actin bundles. ${ }^{10}$ The actin-bundling activity of fascin and its localization in cells are regulated by phosphorylation of serine 39 within the $\mathrm{N}$-terminal actin-binding domain of fascin. ${ }^{14}$ An extracellular component, fibronectin, activates protein kinase $C$ alpha which binds with Ser-39-phosphorylated fascin. This interaction of phosphorylated fascin contributes to cell migration by altering the balance between assembly of fascin protrusions and focal adhesions. ${ }^{13,15}$

Fascin is reported to be over-expressed in carcinomas of colon, pancreatic, breast, lung, esophagus, stomach, skin, and ovary. In addition, up-regulation is also reported in neoplasms like Hodgkin's lymphoma, multiple myeloma, immunocompromised conditions like AIDS and also in salivary gland carcinomas. ${ }^{7,9,16,17}$ Shimamura et al. in 2011 reported the up-regulation of fascin occurring early in the progression from normal epithelium to invasive OSCC ${ }^{18}$ In light of these findings, we conducted a retrospective study on different grades of OED and OSCC.

The aims of our study are as follows: (a) to assess and compare the immunohistochemical expression of fascin in NOM and OED and (b) to correlate the immunohistochemical expression of fascin in different grades of OSCC.

\section{Materials and Methods}

The present retrospective study was conducted at the Department of Oral Pathology and Microbiology, Annoor Dental College and Hospital, Kerala. An Ethical Committee clearance was obtained from our Institutional Review Board to conduct the present study.

This study involves the use of formalin-fixed paraffin-embedded tissues of 10 NOM, 15 OED, and 30 OSCC retrieved from our departmental archives. OSCC specimens were further subdivided into 10 cases each of well (WDSCC), moderate (MDSCC), and poorly differentiated (PDSCC) on the basis of Broder's histopathological grading..$^{19}$ Oral epithelial dysplasia specimens consist of 5 cases each of mild, moderate, and severe dysplasia, which were classified according to WHO 2005 classification. ${ }^{6}$ Normal oral mucosal specimens used for this study were collected during extractions for orthodontic treatment, frenectomies and surgical removal of impacted teeth. Carcinomas of sites other than oral cavity proper like oropharynx and maxillary sinus and NOM from individuals with long-standing tobacco-related habits were excluded in this study. Normal lymph node sections from radial neck dissection were used as positive controls for fascin (Fig. 1A).

\section{Immunohistochemistry}

Paraffin-embedded tissue blocks were cut into 4- $\mu$ m-thick sections and taken onto Superfrost Excel adhesive-coated microscopic slides (Thermo Fisher Scientific, USA). Two sections were obtained from each tissue blocks. The sections were then deparaffinized and rehydrated through xylene and descending grades of alcohol. Thereafter, the sections were placed in EDTA solution inside a commercial microwave for antigen retrieval $(\mathrm{pH}-9.0)$ at $95^{\circ} \mathrm{C}$ for 3 cycles of 5 minutes each. After rinsing twice in Tris buffer saline, the sections were treated with peroxidase block consisting of $3 \% \mathrm{H}_{2} \mathrm{O}_{2}$ in water for 10 minutes to block the endogenous peroxidase activity, followed by a 30-minutes power block to obstruct any nonspecific antigenic sites. The sections were then incubated for 1 hour at room temperature with fascin monoclonal antibody (Biogenex, USA). Super enhancer reagent was added after this step and kept at room temperature for 30 minutes. After washing with Tris buffer saline, the sections were then incubated with one-step polymerHRP reagent for 30 minutes. Freshly prepared diaminobenzidine tetrahydrochloride was used as chromogen for visualization. The slides were counterstained with Harris hematoxylin for 1 minute. Sections were finally cleared and mounted with DPX.

\section{Interpretation of Staining}

The slides were evaluated for immunostaining and scored as positive or negative. Cells showing any cytoplasmic immunoreactivity were considered positive for fascin expression.

To score the fascin expression in OED, four original categories based on the level of epithelial staining were considered. ${ }^{18}$ The expression was scored as

Score 0-if no expression was observed in any part of the epithelium;

Score 1-if expression was restricted to one-third of the epithelium beginning in the basal layer;

Score 2-if expression was observed in two-thirds of the epithelium beginning in the basal layer;

Score 3-if expression was observed in all areas of the epithelium.

Score was calculated in 10 high power fields in each slide and mean was calculated per slide.

The OSCC samples were subjected to evaluation for the intensity of staining and percentage score. ${ }^{20}$ In those cases with staining heterogeneity, the expression was scored according to the predominant staining intensity. It was scored as

Score 0 -negative staining;

Score 1-mild staining;

Score 2-moderate staining; and

Score 3-intense staining.

For percentage score, the number of cells stained were assessed and scored as:

Score 1 -less than $10 \%$ stained;

Score 2-11-50\% stained;

Score 3-51-80\% stained, and

Score 4 -more than $81 \%$ stained.

Score was calculated in 10 high power fields in each slide, and mean was calculated per slide. Final score was obtained by adding percentage score and staining intensity score, which can range from 0 to 7 . Scores from 0 to 2 are considered negative, and scores from 3 to 7 are considered positive. ${ }^{20}$ All the interpretations were carried out by three observers in order to eliminate inter-observer bias.

Data obtained were tabulated, and statistical analysis were carried out using one-way ANOVA and Tukey's post hoc test with the help of SPSS software for windows 22.0.0 and Minitab version 17.1.0 software package.

\section{Results}

\section{Fascin Expression in NOM and OED}

In sections of NOM, 5 out of 10 cases showed negative staining (score 0 ). In the remaining cases, staining was mainly concentrated in the basal or parabasal cell layers (score 1 or 2). To be precise, out of the 5 immunopositive sections of NOM, 3 sections showed staining in the one-third of the epithelium (score 1) and 2 sections showed staining in the two-thirds of the epithelium (score 2) (Figs 1B and C). 

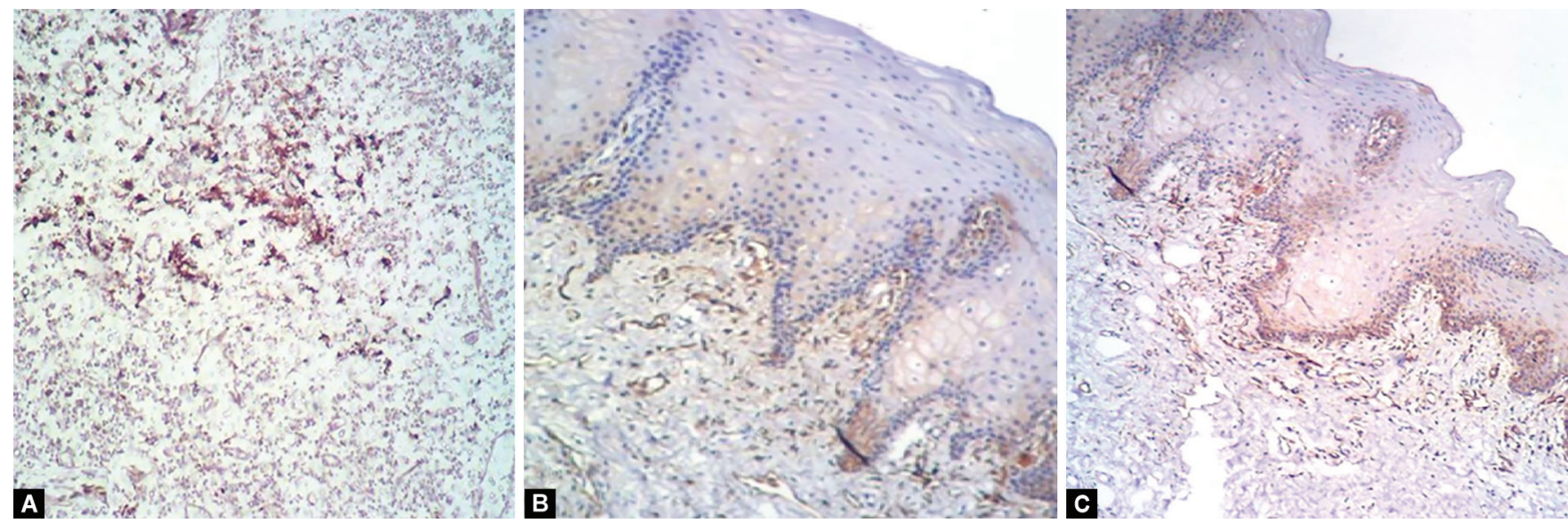

Figs 1 A to C: (A) Photomicrograph of fascin immunostaining in positive control—normal lymph node section (IHC, 40X); (B) Photomicrograph of fascin immunostaining in NOM—score $0(\mathrm{IHC}, 40 \times)$; (C) Photomicrograph of fascin immunostaining in NOM—score 1 (IHC, 40X)
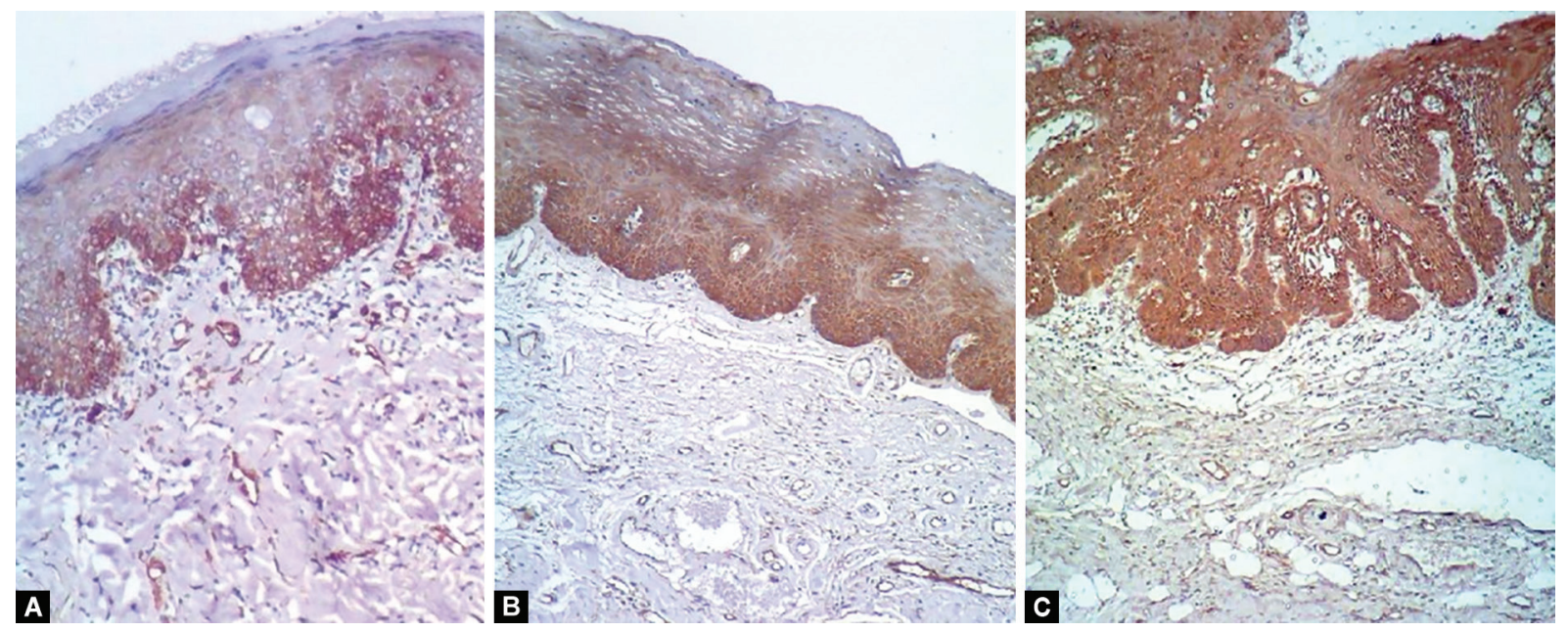

Figs 2A to C: (A) Photomicrograph of fascin immunostaining in mild dysplasia (IHC, 40X); (B) Photomicrograph of fascin immunostaining in moderate dysplasia (IHC, 40X); (C) Photomicrograph of fascin immunostaining in severe dysplasia (IHC, 40X)

Table 1: Immunohistochemical expression patterns of fascin in different grades of OED

\begin{tabular}{llllllll}
\hline IHC score & & 0 & 1 & 2 & 3 & $N$ & Mean \pm SD \\
\hline OED & Mild dysplasia & 2 & 1 & 1 & 1 & 5 & $1.20 \pm 1.30$ \\
& Moderate dysplasia & 0 & 1 & 1 & 3 & 5 & $2.40 \pm 0.89$ \\
& Severe dysplasia & 0 & 0 & 1 & 4 & 5 & $2.80 \pm 0.44$ \\
Total & & 2 & 2 & 3 & 8 & 15 & \\
\hline
\end{tabular}

In a total of 15 cases of OED, except two cases of mild dysplasia, all remaining sections showed immunopositivity for fascin (Fig. 2). To be precise, 2 cases showed no staining in any part of the epithelium (score 0 ); 2 cases showed positivity restricted to one-third of the epithelium (score 1); 3 cases showed positivity in two-thirds of the epithelium (score 2); and 8 cases showed immunohistochemical expression in all areas of the epithelium (score 3). The distribution of fascin immunopositivity in three groups of OED is represented in Table 1.

The mean \pm SD is seen to increase from mild $(1.20 \pm 1.30)$ to severe dysplasia $(2.80 \pm 0.44)$ among the subgroups of OED (Table 1 and Fig. 3). Also Tukey's post hoc test showed a strong statistical significance in fascin expression between NOM and
OED $(p=0.002)$ at $5 \%$ interval (Table 2$)$. The immunohistochemical expression of fascin in OED cases is statistically higher when compared to NOM specimens.

\section{Fascin Expression in OSCC}

Thirty samples of OSCC were taken for the present study, which were further subdivided into 10 each of well, moderate, and poorly differentiated OSCCs. Though all the cases showed positivity for fascin, 4 cases expressed positivity in less than $10 \%$ of cells.

With regard to percentage score, 18 OSCC cases showed score 4 ( $>80 \%$ of positive cells), which includes 5 well, 6 moderate, and 7 poorly differentiated OSCCs. Five cases of OSCC showed score 3 (51-80\% positive cells) and 4 cases showed score 2 (10-50\% 


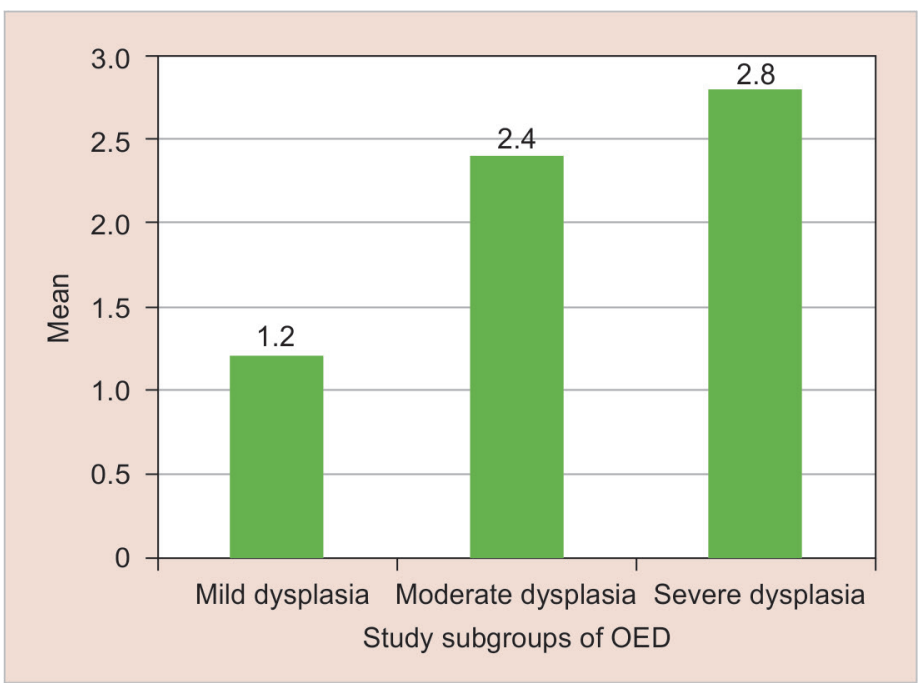

Fig. 3: Increasing expression of fascin in different grades of dysplasia

Table 2: Comparison of fascin expression patterns in NOM and OED (Tukey's post hoc test)

\begin{tabular}{lllll}
\hline Study groups & $N$ & Mean \pm SD & S.E. & p value \\
\hline NOM & 10 & $0.70 \pm 0.82$ & 0.384 & 0.002 \\
OED & 15 & $2.13 \pm 1.12$ & & \\
\hline
\end{tabular}

positive cells). For the intensity score, 12 samples obtained score 3 (intense staining), which includes 3 well, 4 moderate, and 5 poorly differentiated OSCCs. However, 15 samples also reported to show score 2 (moderate staining) comprising 5 well, 6 moderate, and 4 poorly differentiated cases (Fig. 4). The final scores were obtained by summing up percentage score and intensity score. In the present study, all 30 cases had a final score above 3 , which denotes a strong positive immunostaining of fascin in OSCC.

One-way ANOVA test denotes an increased mean \pm SD from well $(5.1 \pm 1.37)$ to poorly differentiated OSCC $(6 \pm 1.24)$ (Table 3 and Fig. 5). Also, the statistical analysis between the subgroups of OSCC using Tukey's post hoc test denotes poor statistical significance between the subgroups (Table 4). The immunohistochemical expression of fascin in different grades of OSCC is seen to be increasing with decreasing grades of OSCC.

\section{Discussion}

The increase in mortality rate of oral cancer is related to the late diagnosis of this devastating disease. The symptoms of oral cancer often presents very late, and sometimes even after the metastasis have occurred. Therefore, the identification of potentially malignant tumors is crucial and important in the diagnostic implications of oral cancer.

In the present study, OED is compared against NOM for fascin expression. Except 2 cases, all other specimens of OED stained positive for fascin. Of the 10 NOM cases, the fascin expression was either negative or concentrated in the basal or parabasal layers. Ergo, it was clearly demonstrated in our study that a statistically significant difference was obtained when the fascin expression of NOM and OED were compared $(p=0.002)$. This was in accordance with the study conducted by Shimamura et al., which demonstrates the expression of fascin in benign epithelial diseases, dysplastic lesions, and oral carcinoma. ${ }^{18}$ In the present study, fascin expression were seen to be increasing with increasing grades of dysplasia (mild to moderate to severe) (Table 1). Therefore, fascin over-expression in epithelial dysplasia suggests a potential role of this protein in the early stages of carcinogenesis. The possibility of fascin to be used as a reliable diagnostic biomarker is thus proved.

A prognostic biomarker is as important as a diagnostic biomarker because it determines the treatment outcome and survival rate of patients. The treatment of oral cancer includes surgery along with chemotherapy or radiotherapy as adjuncts. However, the occurrence of second primary tumors still remains the reason for increasing mortality rates. The prognosis of OSCC is directly proportional to the clinical and histopathological staging. Therefore, the possibility of a potential prognostic marker for determining the malignant tumor behavior and treatment outcome is of great importance.

Several studies involving fascin have been conducted in head and neck carcinomas to investigate its role in tumor progression and invasion. Most of these reports elucidate the importance of fascin in the molecular mechanism of cell motility. During carcinogenesis, invasive cancer cells develop actin-based cell protrusions, which aid in cell migration. ${ }^{21,22}$ Rodigrues et al. elaborated that the knockdown of fascin in OSCC cells promoted cell adhesion and inhibited migration, invasion, and epithelialmesenchymal transition, while the down regulation of fascin can lead to reduced filopodial formation. ${ }^{23}$ Lee et al. reported that in OSCC cell lines, fascin expression is found to have a negative correlation with cell adhesion protein E-cadherin. ${ }^{20}$ In addition, the studies conducted on head and neck carcinomas have reported an association between increased expression of fascin and lymphatic metastasis. ${ }^{24,25}$ The speculations of all these studies shed light to the tumorigenic role of fascin in human oral carcinoma, which implies its significance as a prognostic biomarker.

Our study demonstrated a strong staining in $86 \%$ of OSCC cases. Similar studies conducted by Lee et al. in 46 OSCC cases reported a moderate-to-strong staining in $56.5 \%$ of cases while negative-to-weak staining was seen in $43.5 \%$ of cases. ${ }^{20}$ Out of 131 samples of OSCC studied by Alam et al., only $74.80 \%$ of the cases showed fascin staining while it was not detected in $25.19 \%$ of the cases. ${ }^{26}$ In a study by Chen et al. in 106 cases of tongue 

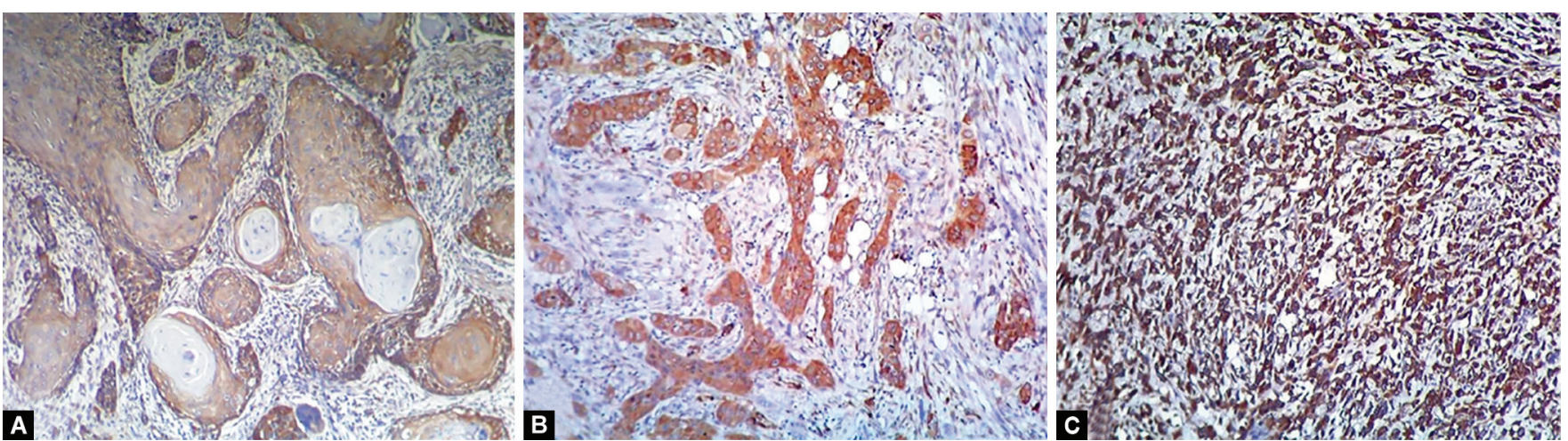

Figs 4A to C: (A) Photomicrograph of fascin immunostaining in WDSCC (IHC, 40X); (B) Photomicrograph of fascin immunostaining in MDSCC (IHC, $40 \times)$; (C) Photomicrograph of fascin immunostaining in PDSCC (IHC, 40X)

Table 3: Immunohistochemical expression scores of fascin in OSCC (one-way ANOVA)

\begin{tabular}{llllll}
\hline Study groups & & $N$ & Mean $\pm S D$ & Fvalue & pvalue \\
\hline OSCC & WDSCC & 10 & $5.1 \pm 1.37$ & 1.1607 & 0.3284 \\
& MDSCC & 10 & $5.6 \pm 1.35$ & & \\
& PDSCC & 10 & $6 \pm 1.24$ & & \\
\hline
\end{tabular}

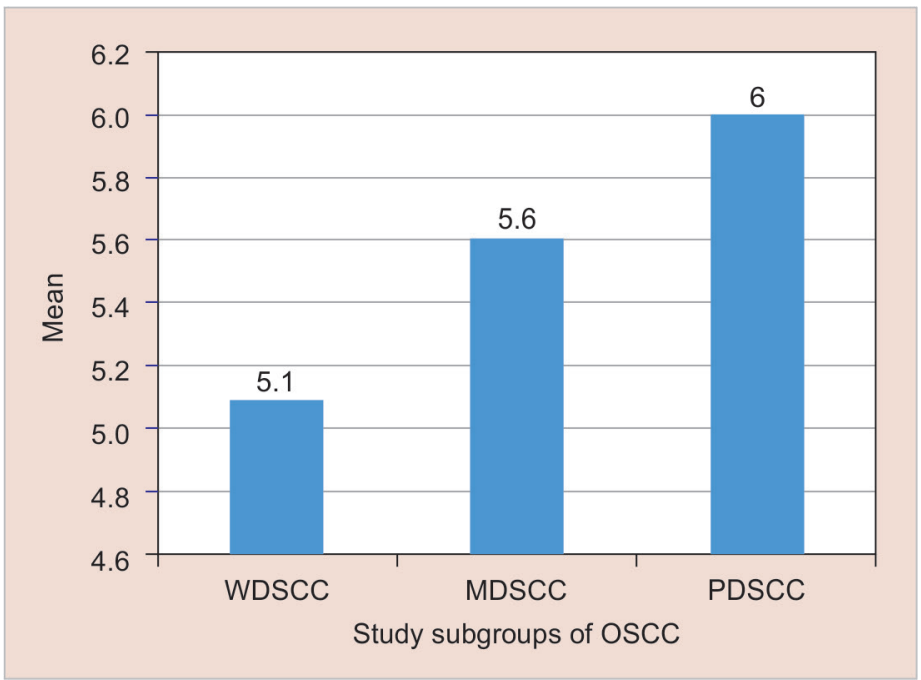

Fig. 5: Expression of fascin in different grades of OSCC

Table 4: Comparison of fascin expression between subgroups of OSCC (Tukey's post hoc test)

\begin{tabular}{lllll}
\hline \multicolumn{2}{l}{ Study subgroups } & Mean difference & pvalue & \\
\hline WDSCC & MDSCC & 0.5 & $>0.05$ & Nonsignificant \\
WDSCC & PDSCC & 0.9 & $>0.05$ & \\
MDSCC & PDSCC & 0.4 & $>0.05$ & \\
\hline
\end{tabular}

carcinoma $62.2 \%$ of cases exhibited high fascin expression, while $34.9 \%$ of cases showed relatively low fascin. ${ }^{27}$ This disparity of fascin immunoreactivity between studies could be attributed to the difference in sample size, the methods of evaluation of staining, or technical errors. Alam et al. and Chen et al. also demonstrated that fascin expression is higher in poor/moderate OSCC than WDSCC. ${ }^{26,27}$ In our study, fascin expression seems to be increasing from well to moderate to poor OSCC (Table 3 and Fig. 5). The aggressiveness of the tumor pertains to the over-expression of fascin and therefore a poorer prognosis.

Fascin expression was seen as (a) diffuse or (b) focal staining in many of the well and moderately differentiated OSCCs under study. The expression was negative in the center while more intense staining was seen toward the periphery of keratin islands (Fig. 4A). This could be due to the fact that the central cells have undergone terminal differentiation and degenerative changes.

Prognostication of carcinoma is essential, but the early diagnosis of potentially malignant disorders proves to be the foundation of successful treatment of any cancer. The limitation of our study includes small sample size of both OED and OSCC. Therefore, further studies are required to analyze the correlation between expression of fascin and histological grades of dysplasia and carcinoma in a larger sample of cases. Also, the chances of fascin to be used as a therapeutic target should be explored more thoroughly. 


\section{Conclusion}

Our study concludes that fascin is over-expressed in both OED and OSCC, which clearly implies that fascin plays a role in carcinogenesis. Also, fascin expression increases with increasing grades of dysplasia, thereby making it an excellent biomarker for identification of premalignancy. The present study also demonstrates that fascin expression increases with decrease in differentiation of OSCC. This could imply that fascin can be used as a prognostic marker for oral carcinoma.

\section{Clinical Significance}

The up-regulation of fascin in dysplasias and carcinomas has been implied to its role in cell transformation and increasing cell motility. Our study reveals positive expression of fascin in OED and OSCC. This implies its use as a diagnostic and prognostic marker in OED and OSCC, respectively.

\section{Acknowledgment}

We acknowledge the help of our technician Mrs Prathiba Basil, MLT, for the processing and sectioning of the specimens.

\section{References}

1. Ghantous Y, Abu IE. Global incidence and risk factors of oral cancer. Harefuah 2017;156(10):645-649.

2. Takiar R, Nadayil D, Nandakumar A. Projections of number of cancer cases in India (2010-2020) by cancer groups. Asian Pac J Cancer Prev 2010;11(4):1045-1049.

3. van der Waal I. Potentially malignant disorders of the oral and oropharyngeal mucosa; terminology, classification and present concepts of management. Oral Oncol 2009;45(4-5):317-323. DOI: 10.1016/j.oraloncology.2008.05.016.

4. Warnakulasuriya S, Ariyawardana A. Malignant transformation of oral leukoplakia: a systematic review of observational studies. J Oral Pathol Med 2016;45(3):155-166. DOI: 10.1111/jop.12339.

5. Holmstrup P, Vedtofte P, Reibel J, et al. Long-term treatment outcome of oral premalignant lesions. Oral Oncol 2006;42(5):461-474. DOI: 10.1016/j.oraloncology.2005.08.011.

6. Warnakulasuriya S, Reibel J, Bouquot J, et al. Oral epithelial dysplasia classification systems: predictive value, utility, weaknesses and scope for improvement. J Oral Pathol Med 2008;37(3):127-133. DOI: 10.1111/j.1600-0714.2007.00584.X.

7. Yamashiro S, Yamakita Y, Ono S, et al. Fascin, an actin-bundling protein, induces membrane protrusions and increases cell motility of epithelial cells. Mol Biol Cell 1998;9(5):993-1006. DOI: 10.1091/ mbc.9.5.993.

8. Zhang FR, Tao LH, Shen ZY, et al. Fascin expression in human embryonic, fetal, and normal adult tissue. J Histochem Cytochem 2008;56(2):193-199. DOI: 10.1369/jhc.7A7353.2007.

9. Vignjevic D, Kojima SI, Aratyn Y, et al. Role of fascin in filopodial protrusion. J Cell Biol 2006;174(6):863-875. DOI: 10.1083/ jcb.200603013.

10. Kureishy N, Sapountzi V, Prag S, et al. Fascin and their roles in cell structure and function. Bioessays 2002;24(4):350-361. DOI: 10.1002/ bies.10070.
11. Zhang $\mathrm{X}, \mathrm{CholH}$, Park JH, et al. Fascin is involved in cancer cell invasion and is regulated by stromal factors. Oncol Rep 2019;41(1):465-474. DOI: 10.3892/or.2018.6847.

12. Lauffenburger $D A$, Horwitz AF. Cell migration: a physically integrated molecular process. Cell 1996;84(3):359-369. DOI: 10.1016/S00928674(00)81280-5.

13. Elkhatib N, Neu MB, Zensen $C$, et al. Fascin plays a role in stress fiber organization and focal adhesion disassembly. Curr Biol 2014;24(13):1492-1499. DOI: 10.1016/j.cub.2014.05.023.

14. Machesky LM, Li A. Fascin: invasive filopodia promoting metastasis. Commun Integr Biol 2010;3(3):263-270. DOI: 10.4161/cib.3.3. 11556.

15. Anilkumar N, Parsons M, Monk R, et al. Interaction of fascin and protein kinase Ca: a novel intersection in cell adhesion and motility. EMBO J 2003;22(20):5390-5402. DOI: 10.1093/emboj/cdg521.

16. Tan VY, Lewis SJ, Adams JC, et al. Association of fascin-1 with mortality, disease progression and metastasis in carcinomas: a systematic review and meta-analysis. BMC Med 2013;11:52. DOI: 10.1186/17417015-11-52.

17. Hashimoto $Y$, Skacel $M$, Adams JC. Roles of fascin in human carcinoma motility and signaling: prospects for a novel biomarker? Int J Biochem Cell Biol 2005;37(9):1787-1804. DOI: 10.1016/j.biocel.2005. 05.004 .

18. Shimamura $\mathrm{Y}, \mathrm{Abe} \mathrm{T}, \mathrm{Nakahira} \mathrm{M}$, et al. Immunohistochemical analysis of oral dysplasia: diagnostic assessment by fascin and podoplanin expression. Acta Histochem Cytochem 2011;44(6):239-245. DOI: 10.1267/ahc.11032.

19. Broders AC. Carcinoma of the mouth: types and degrees of malignancy. Am J Roentgenol Radium Ther Nucl Med 1927;17:90-93.

20. Lee TK, Poon RT, Man K, et al. Fascin over-expression is associated with aggressiveness of OSCC. Cancer Lett 2007;254(2):308-315. DOI: 10.1016/j.canlet.2007.03.017.

21. Eddy RJ, Weidmann MD, Sharma VP, et al. Tumor cell invadopodia: invasive protrusions that orchestrate metastasis. Trends Cell Biol 2017;27(8):595-607. DOI: 10.1016/j.tcb.2017.03.003.

22. Li A, Dawson JC, Forero-Vargas M, et al. The actin-bundling protein fascin stabilizes actin in invadopodia and potentiates protrusive invasion. Curr Biol 2010;20(4):339-345. DOI: 10.1016/j.cub.2009. 12.035 .

23. Rodrigues PC, Sawazaki-Calone I, de Oliveira CE, et al. Fascin promotes migration and invasion and is a prognostic marker for oral squamous cell carcinoma. Oncotarget 2017;8(43):74736-74754. DOI: 10.18632/ oncotarget.20360.

24. Papaspyrou K, Brochhausen C, Schmidtmann I, et al. Fascin upregulation in primary head and neck squamous cell carcinoma is associated with lymphatic metastasis. Oncol Lett $2014 ; 7(6)$ : 2041-2046. DOI: 10.3892/ol.2014.2007.

25. Routray S, Kheur S, Chougule HM, et al. Establishing fascin overexpression as a strategic regulator of neoplastic aggression and lymph node metastasis in oral squamous cell carcinoma tumor microenvironment. Ann Diagn Pathol 2017;30:36-41. DOI: 10.1016/ j.anndiagpath.2017.05.013.

26. Alam H, Bhate AV, Gangadaran P, et al. Fascin overexpression promotes neoplastic progression in OSCC. BMC Cancer 2012;12:32. DOI: 10.1186/1471-2407-12-32.

27. Chen Y, Tian T, Li ZY, et al. FSCN1 is an effective marker of poor prognosis and a potential therapeutic target in human tongue squamous cell carcinoma. Cell Death Dis 2019;10(5):356. DOI: 10.1038/ s41419-019-1574-5. 